\title{
Rapidly Spreading Optic Pathway Glioma with Intracranial Extension - Case Report
}

\author{
Aashish Dhage, B Shukla* and Purendra Bhasin \\ Ratan Jyoti Netralaya, Gwalior, India \\ *Corresponding Author: B Shukla, Department of Ophthalmology, India.
}

Received: May27, 2019; Published: July 15, 2019

DOI: 10.31080/ASNE.2019.02.0080

\begin{abstract}
Optic pathway glioma is a tumor arising from astrocytes and is one of the most common tumors of optic nerve. It comprises of $5 \%$ of pediatric intracranial tumour [1]. Usual presentation is of a slow growing tumor in the first two decades of life. There have been numerous reviews on this subject and one such quote that $60 \%$ of optic pathway gliomas occur in children less than 5 years of age. It can have a range of presenting features starting from vision loss, proptosis, extraocular movement restriction, strabismus, optic disc edema or pallor. Inflammatory signs have not been reported. $30 \%$ of patients with glioma have associated neurofibromatosis type-1. These patients have bilateral presentation and superior prognosis. The tumor has been reported to spread to chiasma (40-75\%) and the diencephalon (33-60\%) but intraocular extension is rarely noted [2]. Histologically, the tumor shows spindle shaped pilocytic astrocytes and glial filaments [3]. Treatment options can range from observation for asymptomatic and slowly growing tumor to surgical resection for vision threatening tumors and tumors causing proptosis. Radiation therapy and chemotherapy combination can be an option.
\end{abstract}

Keywords: Optic Pathway; Optic Nerve; Glioma

\section{Case Report}

We report a case of a 16 month old child who developed a rapidly progressive optic pathway glioma of right eye with widespread intracranial extensions. A 16 month old female child presented to us with complaints of proptosis and deviation of right eye since two and a half months. There was no history of white reflex in the eye, trauma or change in the size of swelling on changing posture. Family history was non-contributory. Parents did not report any history of skin lesions or any other swelling over the body. On examining the child, no dermatological signs of neurofibromatosis type- 1 were found. Assessment of visual behaviour of the child revealed no fixation to light in right eye whereas left eye showed central steady and maintained fixation. Detailed ocular examination showed presence of massive axial proptosis with inferior dystopia [Figure 1]. It was non pulsatile in nature, retropulsion was positive and no inflammatory changes were seen. Extra-ocular movements were limited in abduction and elevation in that eye. Anterior segment examination showed afferent pupillary defect. No other signs of Neurofibromatosis-1, like eyelid neurofibroma or lisch nodules were observed. Fundus examination showed complete optic atrophy with vascular tortuosity around the disc.

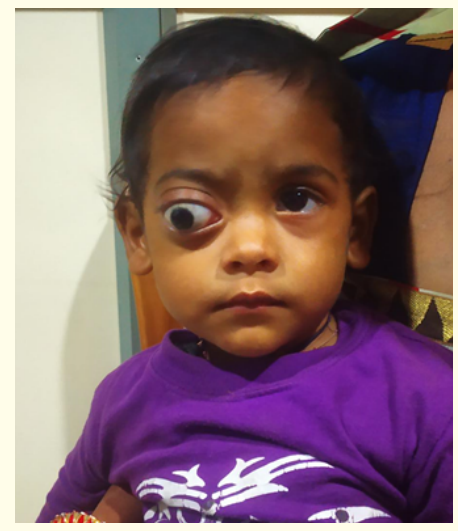

Figure 1

1. Consultant, Department of Pediatric Ophthalmology, Ratan Jyoti Netralaya, Gwalior, India

2. Director of Research and Training, Ratan Jyoti Netralaya, Gwalior, India

3. Director and Chief Consultant, Cataract and Refractive services, Ratan Jyoti Netralaya, Gwalior, India 
Multiple choroidal folds were seen. Left eye examination was normal in all respects apart from absent consensual light reflex. On history and examination we could narrow down our differential diagnosis to optic nerve glioma, lymphangioma, rhabdomyosarcoma and a rare possibility of meningioma [4].

We investigated the child with USG B-scan which showed retrobulbar mass near optic nerve which is pressing the posterior eyeball. Also the mass had cystic spaces [Figure 2A, 2B]. With this information we narrowed our diagnosis to optic nerve glioma and went ahead with neuroimaging. Neuroimaging confirmed our diagnosis and it detailed the extent of the tumour. MRI [Figure 3a, $3 b, 3 c]$ reported an entire bulky optic nerve with peripheral sheet like cystic intensity extending into the optic chiasma. There is also a large cystic mass lesion in the sellar and suprasellar region effacing the adjacent suprasellar system. It is encasing the cavernous segment of internal carotid artery and the origin of middle carotid artery. This mass appeared to be infiltrating into the adjacent brain parenchyma in medial temporal lobes of both sides. Radiologist was of the opinion that it is a case of malignant optic pathway glioma. The child was referred to a neurosurgeon who advised multimodal treatment for this disease.

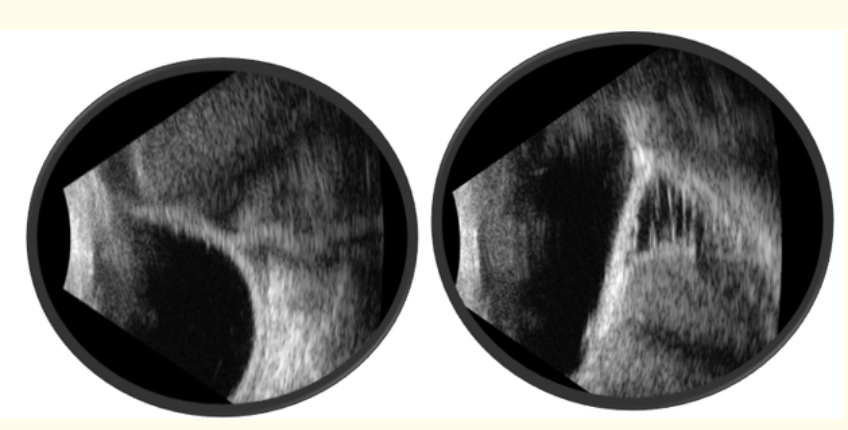

Figure 2A and 2B

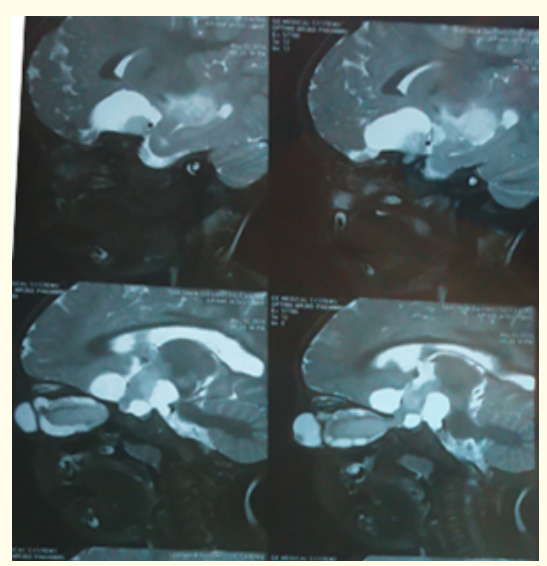

Figure 3a

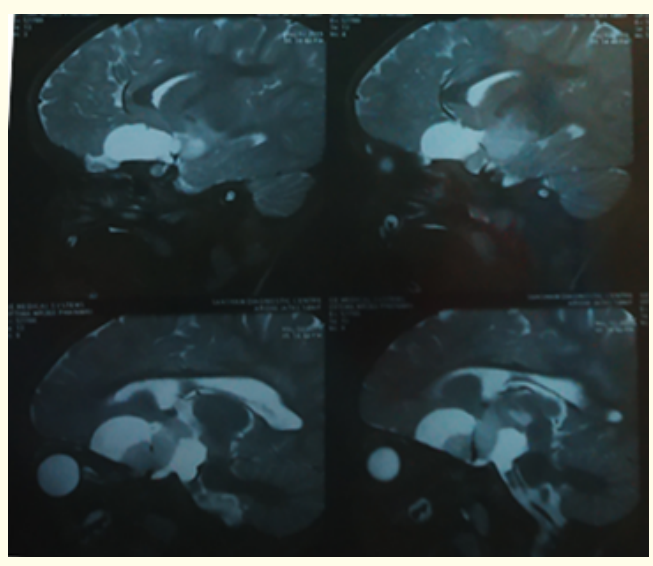

Figure 3b

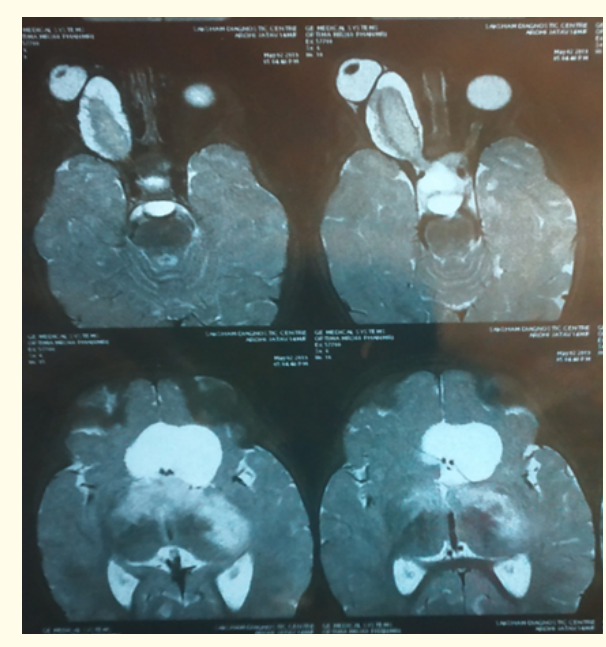

Figure 3c

\section{Discussion}

Optic nerve glioma is in itself a slowly growing tumour. However, this case presented a child just 16 month old with rapidly progressing glioma which could be malignant. Unilateral malignant gliomas are of rare occurrence and difficult to treat. The final diagnosis can only be confirmed after optic nerve biopsy. Our case is significant since the age of onset is very less and progression is rapid. Also malignant transformation is rarely noted in optic nerve glioma. Prognosis is very poor and survival rate is not good [5].

This atypical onset is inconsistent with studies done by North., et al. [6]. and Parsa., et al [7]. as they reported mean age as $10.9 \mathrm{yrs}$. Our presentation of unilateral proptosis with strabismus and loss of vision is similar with findings of Tow., et al [8]. and Sharma., et al [9]. Neuroimaging was crucial in understanding the catastrophic nature of the extent of the tumour and the urgency needed to start on treatment. 


\section{Conclusion}

We conclude that a tumour like optic nerve glioma which has a tendency to be indolent, slow growing and forgiving turned out to be exactly opposite of its documented nature. We have not concluded that the tumor we encountered was of malignant nature with hislological report but the rapidity of the growth points towards a malignant growth. Radiologist also had a similar view of the nature of this growth. Thus all optic nerve growths need to be investigated with caution. This child might not have a good prognosis but henceforth an optic nerve glioma should be looked at with suspicion.

\section{Acknowledgement}

We are thankful to Mr. Kapil Patel for secretarial help.

\section{Bibliography}

1. Janss AJ., et al. "Optic pathway and hypothalamic/chiasmatic gliomas in children younger than age 5 years with a 6 -year follow-up". Cancer 75 (1995): 1051-1059.

2. Moosumeh Eghtedari., et al. "Optic nerve glioma with complete intraocular extension". Journal of Current Ophthalmology 28 (2016): 228-231.

3. Kanski JJ., et al. "Clinical ophthalmology: A systematic approach (7th ed.)". Edinburgh New York: Elsevier/Saunders (2011).

4. Dutton JJ. "Optic nerve sheath meningiomas". Ophthalmology 37 (1992): 167-183.

5. Wabbels B., et al. "Unilateral adult malignant optic nerve glioma". Graefe's Archive for Clinical and Experimental Ophthalmology 242.9 (2004): 741-748.

6. North K., et al. "Optic gliomas in neurofibromatosis type 1: role of visual evoked potentials". Pediatric Neurology 10 (1994): 117-123.

7. Parsa CF., et al. "Spontaneous regression of optic gliomas: thirteen cases documented by serial neuroimaging". Archives of Ophthalmology 119 (2001): 516-529.

8. Tow SL., et al. "Long-term outcome in children with gliomas of the anterior visual pathway". Pediatric Neurology 28 (2003): 262-270.

9. Sharma A., et al. "Case report: haemorrhagic changes in pilocytic astrocytoma of the optic nerve". Orbit 9 (1990): 29-33.

Volume 2 Issue 8 August 2019

(C) All rights are reserved by B Shukla., et al. 